\title{
Path completion after haptic exploration without vision: Implications for haptic spatial representations
}

\author{
ROBERTA L. KLATZKY \\ Carmegie Mellon University, Pittsburgh, Pennsylvania
}

\begin{abstract}
Subjects haptically explored two legs of a triangular path and responded by returning to the origin. Seven conditions were tested, varying in (1) whether the path was imaginally displaced between the initial exploration and the response; (2) the nature of the displacement, if present-rotation or translation; (3) variability in the origin location across trials; and (4) instructions to complete a triangle versus remembering the origin location. Mean distance and angle responses were modeled by the encodingerror model (Fujita, Klatzky, Loomis, \& Golledge, 1993), which attributes errors to misencoding of the path legs and angle. The model failed to predict the finding of systematic errors in response distance but not response angle, a dissociation that held when the path was undisplaced or imaginally translated. Rotation before responding produced errors more consistent with the model. The data suggest use of a body-centered representation to complete undisplaced or imaginally translated paths, but adoption of an object-centered representation after imagined rotation, as is more consistent with pathway completion using whole-body locomotion.
\end{abstract}

This paper is concerned with people's representation of the positions of objects in planar space that they explored haptically, without vision. In terms defined by Lederman, Klatzky, and associates (Lederman, Klatzky, Collins, \& Wardell, 1987), it is intended to investigate the representation of manipulatory space, which lies within arm's reach; however, the present study was motivated by research on what Lederman et al. called ambulatory space, which is explored by locomotion. A central concern is whether processing mechanisms that are applied during locomotion without vision will also be applied to haptic spatial perception within the plane of a tabletop. The processing mechanisms of interest are those described by what is called the encoding-error model (Fujita, Klatzky, Loomis, \& Golledge, 1993), discussed below.

Lederman et al. (1987) demonstrated that similar processes can be used for perception of distance in both manipulatory and ambulatory space. Subjects in their experiments explored two legs of a triangle either manually or on foot, and then estimated the length of the third leg. In both cases, the estimate increased with the length of the first two legs, suggesting that subjects based the estimated length of the third leg on the distance that the limbs moved. Apparently, this "movement-based heuristic" applied regardless of scale or means of exploration, manual or locomotor.

Despite this evidence for possible similarities, there are differences between manual and locomotor exploration

Correspondence should be addressed to R. L. Klatzky, Department of Psychology, Carnegie Mellon University, Pittsburgh, PA 15213-3890 (e-mail: klatzky@cmu.edu). that seem likely to affect spatial processing. An obvious difference is that the two types of exploration occur at different scales. Another important difference, which is emphasized here, is that with manual exploration, the subject's stationary body provides a reference for the locations of touched points in space. But because the ambulatory traveler moves the entire body through space, his/her position with respect to stationary reference locations is constantly changing. This access to fixed reference locations during manipulation, but not locomotion, is likely to be a critical difference in tasks that require subjects to indicate the locations of points in space. One such task, which is the basis for the present research, is called path completion.

In performing path completion in ambulatory space, subjects first walk an outbound path comprising multiple segments, and then attempt to complete the path by returning directly to the origin of travel. They do so in the absence of vision, which would otherwise provide sight of distal landmarks and patterns of optic flow that accompany movement. Lacking such visual cues, subjects in the path-completion task must rely on other sources of information, including velocity and acceleration signals provided by proprioception, or the perception of time or effort. The processing of velocity or acceleration signals to determine changes in location over time is called path integration. Many lower organisms are known to perform path integration well (Etienne, Maurer, \& Séguinot, 1996; Gallistel, 1990; Maurer \& Séguinot, 1995). Studies of humans indicate that they certainly perform nonrandomly when asked to return to the origin of travel, but they also make systematic errors.

The present study was directly patterned after an experiment of Loomis and associates (Loomis et al., 1993) that 
investigated human path integration. Subjects in their experiment were blindfolded and guided along an outbound path consisting of two linear segments (legs) with an angle in between, as shown in Figure 1. These components will be called Leg 1, Leg 2, and Angle 1. Subjects then attempted to walk directly back to the origin, completing a triangle. The third leg of this triangle will be called the response distance, and the angle between Legs 2 and 3 will be called the response angle. In walking back to the origin, the subjects' trajectory yielded observed values of the response distance and response angle. The correct valuesthose that would be obtained if the subject returned to the exact location of the origin and hence completed the triangle - will be called the actual response distance and actual response angle of the path.

When subjects' observed response distance and angle were compared with the actual response distance and angle, a regular pattern was observed: Subjects tended to overrespond when the actual value was low and to underrespond when it was high (e.g., to walk too far if the actual response distance was short and to walk too short a distance when the actual response distance was long). The pattern of overresponding for low actual values and underresponding for high actual values means that the range of subjects' responses was considerably compressed relative to the range of actual values in the stimuli. Subjects' observed responses were most accurate near the mean of actual values, as computed across all the paths used in the experiment. This suggests that subjects learned about the distribution of values as they went through some number of trials.

A model that provided an excellent account of the Loomis et al. (1993) data was developed by Fujita et al. (1993). The model is called the encoding-error model because it attributes the systematic pattern in the errors to the process of encoding the outbound path (Leg 1, Angle 1, Leg 2). Specifically, the model divides processing in the path completion task into (1) encoding processes, which culminate in an internal representation of the outbound path; (2) processes that compute the trajectory required to return to the origin of travel; and (3) processes that execute that trajectory. According to the model, all the systematic errors in responding arise in the first set of processes, which constitute encoding. No systematic error arises in computing the response trajectory from the encoded values or executing that trajectory, although random noise may arise during these processes.

The model was fit to the data of Loomis et al. (1993) by estimating six parameters-namely, the encoded value corresponding to each possible value of leg length and angle in the outbound path. The paths in the experiment were generated from a factorial combination in which Leg 1 and Leg 2 could each take three possible values (2, 4 , or $6 \mathrm{~m}$ ), and Angle 1 could take three possible values

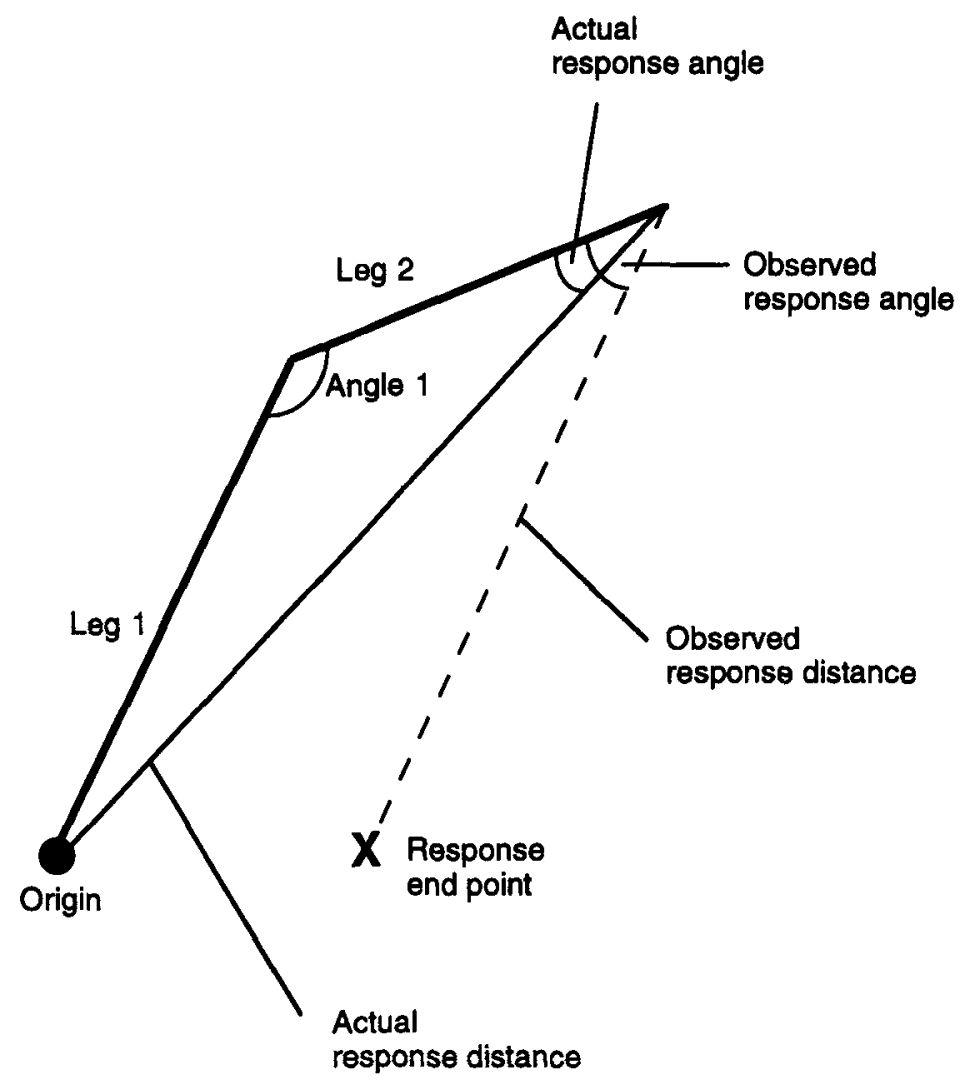

Figure 1. Structure of the path completion task with a triangular stimulus. 
$\left(60^{\circ}, 90^{\circ}\right.$, or $\left.120^{\circ}\right)$ for a total of 27 paths. Thus the model had one parameter corresponding to the encoded version of a leg length of $2 \mathrm{~m}$, one for $4 \mathrm{~m}$, and one for $6 \mathrm{~m}$, and, similarly, a parameter corresponding to the encoded version of a $60^{\circ}$, a $90^{\circ}$, and a $120^{\circ}$ angle. The estimation procedure chose a set of parameters, made a prediction for each path on the basis of those parameters, and then determined the error in prediction. Each path was evaluated in several steps, as follows: First, describe the encoded equivalent of the path. That is, use the current parameter set to assign encoded values corresponding to the actual value of Leg 1, Leg 2, and Angle 1 in the path. It is this step of assigning encoded values that introduces systematic error into the path representation. Second, apply trigonometric formulae to the encoded values to compute what the observed response distance and observed response angle would be. This computation is done without error; that is, the hypothetical subject is assumed to compute the response trajectory perfectly, but from erroneously encoded stimulus values. Third, assume that the response distance and angle are executed without error and predict where in space the subject would have stopped when attempting to complete the path. Finally, compute a measure of model error, which is the Euclidean distance between the predicted stopping point and the empirically observed stopping point for the path. This error was ultimately summed over the 27 paths to determine the predictive value of the given parameter set. This sequence of steps was repeated for a new set of parameters until a parameter set was identified that minimized the model error.

Two functions were then plotted. One related the modeldetermined parameter for each leg length to the actual value; the other related the model-determined parameter for each angle to the actual value. Both functions proved to be highly linear. This allowed Fujita et al. (1993) to reduce the number of parameters in the model from six, corresponding to the encoded value for each possible actual value, to four, corresponding to the slope and intercept of the function relating encoded values to actual values. The encoding functions were as follows (where $d$ stands for distance in meters and $a$ stands for angle in degrees, and the prime indicates the encoded value):

$$
\begin{aligned}
& d^{\prime}=.60 d+1.2 \\
& a^{\prime}=.48 a+50
\end{aligned}
$$

The fact that the slope of the encoding function is less than 1 means that subjects tended to overestimate the lowest value of leg length or angle in the path and to underestimate the highest value. The encoding functions crossed the diagonal near the middle value, indicating that it was encoded accurately. This pattern in the encoding functions is similar to the pattern found when the observed response angles and observed response distances were compared with the actual values. That is, there tended to be overresponding to low actual values, underresponding to high actual values, and accurate responding near the mean of actual values. According to the model, the pattern in the observed responses derives from the similar pattern in the underlying encoding functions.

The model was able to provide an excellent fit to these systematic error patterns in the triangle-completion data of Loomis et al. (1993). Application of the model to subsequent data sets (Klatzky, Beall, Loomis, Golledge, \& Philbeck, in press) replicated the general trends for the encoding functions to be linear with positive intercepts and slopes less than 1.0. However, the encoding functions obtained from other data sets did not necessarily exhibit most accurate encoding at the mean of actual stimulus values, nor did the function relating responses to actual values generally cross the diagonal near the mean. (For a review of other aspects of the model, see Klatzky, Loomis, \& Golledge, 1997.)

The representation assumed by the encoding-error model is what has been called object-centered (Marr, 1982). The location of the path in surrounding space is, according to the model, irrelevant. What underlies systematic error is the encoding of leg and angle values, not their position in externally defined space. The idea that subjects did not consider the position of the path in space is not surprising given that they performed repeated trials without access to distal features. Lacking external spatial referents, they were forced to encode locations on the path, such as the origin, relative to other points within the path itself. ${ }^{1}$ As noted, a crucial difference between path completion via locomotion and path completion via manipulation is that in the latter case, the subject's body remains fixed while the path is explored. The stability of the body allows the location of the origin to be coded relative to haptically accessible features external to the pathway itself (e.g., the sagittal plane of the body or the table edge).

A principal question addressed in this paper was whether, given this difference in possible reference systems, path completion in manipulatory space could be described by the encoding-error model. Efforts to address this question took several forms. Subjects' performance in the tabletop path-completion task was examined to determine whether the same patterns of systematic error that were previously observed with navigation on foot would be observed with manual exploration. An important aspect of the previous results is that similar errors were found in both components of the observed response, angle, and distance. This is consistent with the assumption of the encoding-error model that the two response components are computed from a common set of encoded values. If one measure is erroneous, the other should be, too. To further determine its applicability, the encoding-error model was fit to the data in order to quantitatively assess whether the systematic errors could be accounted for by misencoding the parameters of the outbound path.

Other sources of error are known to occur in manipulatory space. As noted, people may make use of heuristics, such as basing the estimate of the Euclidean distance between points on the length of an indirect path between them. Because this type of error arises in computing the return trajectory, the use of such a heuristic is contrary to 
the encoding-error model's assumption that all error arises in encoding the outbound path. Other demonstrated sources of haptic perceptual error take the form of anisotropies. One, the oblique effect, affects perception of line orientation; there are higher errors in determining the orientation of lines that are oblique than those that are oriented along a horizontal or vertical axis (e.g., Appelle \& Countryman, 1986; Gentaz \& Hatwell, 1995). A second anisitropy, the radial/tangential effect, relates to distance perception; motions that are radial relative to the body are overestimated in extent relative to motions that are tangential to the body (see, e.g., Cheng, 1968; Marchetti \& Lederman, 1983). However, the outbound and return trajectories in the present study occurred over a substantial range of orientations and involved both radial and tangential movements so that these tendencies would not be expected to have a systematic relation to the path parameters.

In addition to examining level and source of error in the path-completion task, other manipulations were meant to further address the reference system that was used to code the location of the origin. In the basic version of the task, the subject completed 27 paths, all originating at the same point on the tabletop, which was also the location of the correct response. In a contrasting condition, the paths originated at different points from trial to trial. This condition was included to determine whether subjects learned to return to a common spatial location, if one was available. Such spatial learning would be inconsistent with the model's assumption that points on the path-including the originare encoded in an object-centered system, not relative to path-external referents. Another condition in this study explicitly encouraged coding in terms of referents outside the path by informing subjects that a single common origin had been used and instructing them to try to return to its location after the outbound movement along the path.

Two other conditions assessed the vulnerability of performance to imagined displacement of the path before responding. The displacement was either a linear translation or rotation. In the translation condition, the subject moved his/her finger leftward, parallel to the edge of the table, at the end of the second leg, before making the response. In the rotation condition, the finger was rotated $45^{\circ}$ at the end of the second leg. In both cases, subjects were instructed to respond as if the path had been displaced along with the finger, and accordingly, to move to the displaced origin that would result.

The inclusion of both translation and rotation displacements in this study was motivated by research on navigation in ambulatory space, which indicates that updating one's angular position relative to objects in space is easier after an imagined translation of the body than it is after an imagined rotation (Rieser, 1989). The task in those studies was to adopt an imagined position in space (specified by location and orientation) that would result from translation or rotation and then report the directions of known objects from that new position. In the present task, people did not imagine themselves translating or rotating; rather, they imagined the displacement of the pathway that would be equivalent to their finger motion. If rotation is still more disruptive than translation, it would suggest that imagined displacement of an object relative to the body (as in this study) requires processing similar to imagined displacement of the body relative to an object.

\section{METHOD}

\section{Subjects}

The subjects were 64 right-handed Carnegie Mellon University students. They participated to satisfy a course requirement or for pay. They were assigned to one of seven conditions (title of condition is indicated in parentheses): (1) no translation before responding, with 1 starting point (no-trans/1); (2) no translation before responding, with 10 starting points used across the pathways (no-trans/10); (3) 4-cm translation before responding, with 1 starting point (trans/1); (4) 4-cm translation before responding, with 10 starting points (trans/10); (5) $9.5-\mathrm{cm}$ translation, which used 10 starting points (large-trans); (6) rotation before responding, which used 10 starting points (rotation); and (7) instructions to return to the spatial location of the origin, which used a single starting point (spatiallocation). There were 8 subjects in each condition except trans $/ 1$, which used 16. The larger $n$ was tested in the trans/ 1 condition because it was initially of particular interest for fitting the model, being potentially most similar to conditions used in the ambulatory studies. Specifically, in the trans/ 1 condition, the translation before responding precluded coding the position of the origin relative to the body at the start of the trial, and the constant starting point maximized the potential for learning. However, the model solution was quite stable across similar conditions, and null effects of some variables ultimately allowed the model to be fit to pooled data, as described below.

\section{Stimuli and Procedure}

The subject's task was to move his/her index finger along a path consisting of two linear segments (legs) connected at an angle, and then return to the starting point, in the absence of vision. The path was made of raised dots approximately $2 \mathrm{~mm}$ in diameter, which were pressed into Braillon paper and then inverted. The start and end of the path were marked with a sandpaper dot $5 \mathrm{~mm}$ in diameter. The path was oriented so that the first leg was at an angle of $70^{\circ}$ relative to the subject's frontal plane; the second leg of the path always continued toward the right. This positioning allowed for comfortable arm orientation during right-handed exploration. Twenty-seven paths were formed by the factorial combination of three variables: length of first leg, length of second leg (both 4,8 , or $12 \mathrm{~cm}$ ), and angle between legs $\left(60^{\circ}, 90^{\circ}\right.$, or $\left.120^{\circ}\right)$. The paths were presented in random order for each subject, preceded by one demonstration path and two practice paths.

An answer sheet was also prepared, consisting of a single sandpaper dot placed on a braillon sheet in a location corresponding to the path end point. In the translation conditions, the answer sheet also contained a finely dotted line, which started at the sandpaper dot and proceeded leftward parallel to the subject's frontal plane, until it terminated in another sandpaper dot. In the rotation condition, two finely dotted $2-\mathrm{cm}$ lines intersected at the sandpaper dot and formed a $45^{\circ}$ angle; one of these lines was aligned with the second leg of the path. These conditions are illustrated in Figure 2.

Before beginning the experimental trials, the subject was given 6 min to complete a set of mental rotation problems. The subject was then blindfolded, and trials with the paths began. On each trial, the experimenter placed the braillon answer sheet on the tabletop and placed the sheet containing the path on top of it so that the path end point lay directly above the sandpaper dot on the answer sheet. The experimenter placed the subject's hand on the starting point, and the subject explored the pathway by proceeding along the dotted line. 

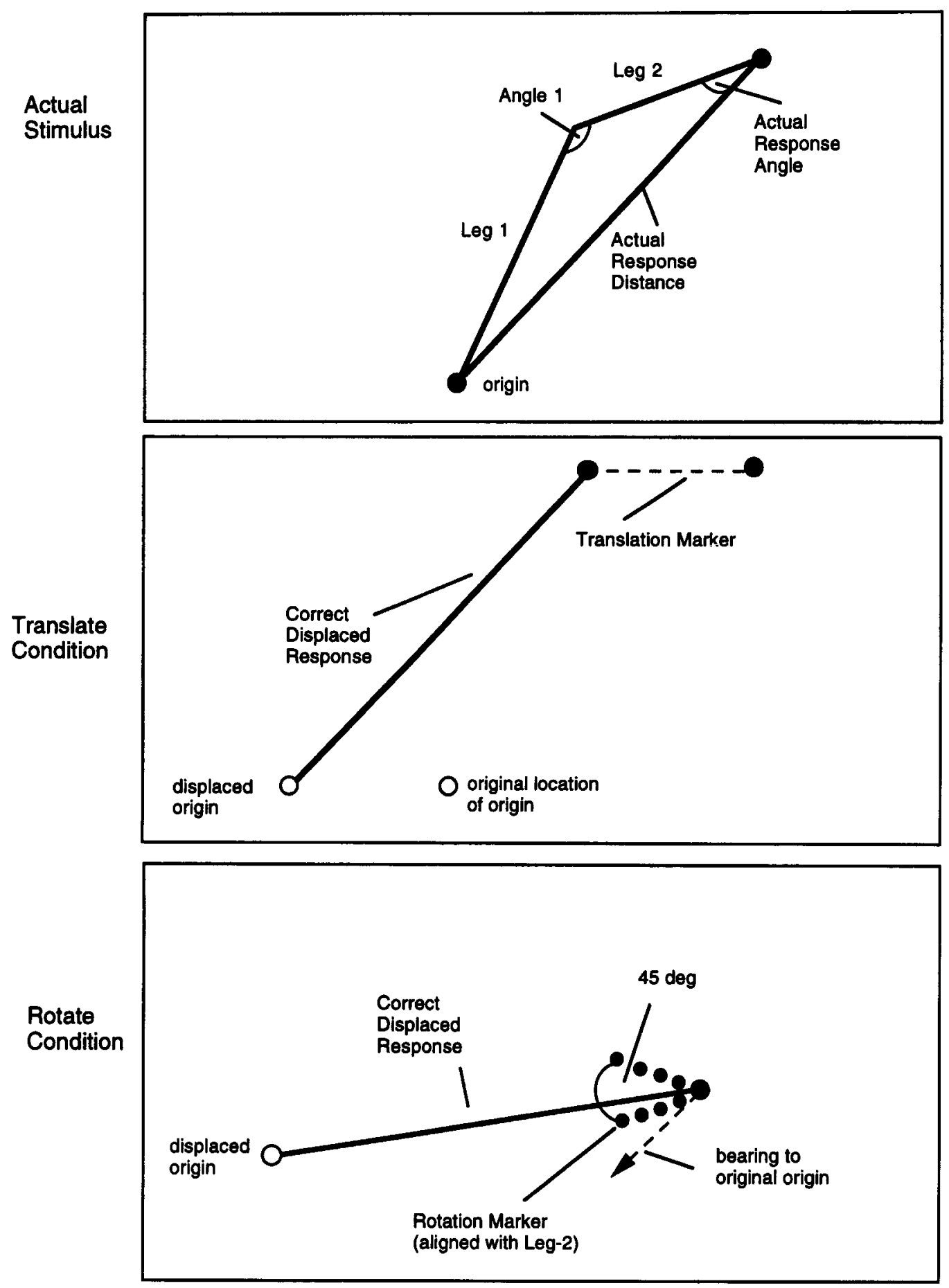

Figure 2. Experimental manipulations. Top panel, typical stimulus and the correct response; the black dots indicate the sandpaper marking the start and end points of the outbound path. The answer sheet, in the absence of translation or rotation, would have the upper dot only. Middle panel, translate condition. The answer sheet would contain the sandpaper (black) dot and translation marker; also shown are the correct response and the original and displaced origins. Bottom panel, rotation condition. The answer sheet would contain the sandpaper (black) dot and rotation marker; also shown are the correct response and displaced origin and the bearing from the point of response to the original origin (along the original Leg 3). 
$\mathrm{He} / \mathrm{she}$ could only move forward on the path and could explore it only once. At the end of the path, the subject raised the finger slightly, allowing the experimenter to remove the sheet containing the path and expose the answer sheet mounted directly beneath it. The subject's finger then fell on the dot on the answer sheet.

In all but the spatial-location condition, subjects were told that the path was the first two legs of a triangle. In the no-trans conditions, they were instructed to respond so as to "go directly back to the origin ... or to complete the triangle." They moved their finger from the sandpaper dot on the answer sheet to an (unmarked) location that they thought corresponded to the position of the origin. In the trans conditions, before responding, the subject first moved the finger leftward along the finely dotted line on the answer sheet until he/she encountered the second dot. The subject was instructed to respond as if the entire path had moved along with the finger and to move to the translated origin that would result from this movement. In the rotation condition, the subject encountered, on the answer sheet, the $45^{\circ}$ angle, which had its apex at the sandpaper dot and had one leg aligned along the second leg of the path. The subject was instructed to think of the aligned leg as if it were the second leg of the path and to rotate the finger clockwise to feel the $45^{\circ}$ angle, and then respond as if the second leg, and along with it the entire path, had rotated with the finger. The subject was then to move to the position of the origin that would result from the rotation. The spatial-location condition was comparable to the no-trans/1 condition, but subjects were explicitly told that the origin would always be in the same place on the tabletop, and the task was to find that location despite having moved the finger along the path. No mention was made of the triangular shape of the path. In all conditions, after moving to the position where the origin was thought to be, the subject rotated the finger slightly so that the experimenter could mark the response position on the answer sheet. No feedback was given.

In all translation conditions but the large-trans condition, the length of the translation (i.e., the length of the line on which the subject's finger moved before the response) was $4 \mathrm{~cm}$. In the large-trans condition, it was $9.5 \mathrm{~cm}$; this value was selected so that the distance between the original origin and the origin after the displacement was the same, on average, as in the rotation condition. Subjects in the large-trans and rotation conditions were shown illustrations like those in Figure 2 to help them understand the task.

The trans/ 1 , no-trans $/ 1$, and location-match conditions all used 1 starting point. The trans $/ 10$, no-trans $/ 10$, rotation, and large-trans conditions used 10 starting points. In the conditions with 1 starting point, all pathways began at a point that lay in the subject's saggital plane and was $30 \mathrm{~cm}$ from the edge of the table. In the conditions with 10 starting points, they were arranged in two staggered rows: Five points were arranged $7.5 \mathrm{~cm}$ apart in a row $30 \mathrm{~cm}$ from the subject and centered on the subject's saggital plane; the remaining 5 starting points lay in an isomorphic row that was $7.5 \mathrm{~cm}$ distal and $3.25 \mathrm{~cm}$ rightward from the first. Each of the 10 starting points was used two or three times over the 27 pathways, with the selection of the starting points otherwise random.

\section{RESULTS}

The data were the observed response distance, defined as the length of the leg traced by the subject, and the $o b$ served response angle, defined as the internal angle in the triangle that was formed by the second leg (translated or rotated, in the relevant conditions) and the subject's observed response leg. These were based on the end point of the subject's response (i.e., the response trajectory was not monitored directly). Figures 3 and 4 show, for the various conditions, the mean response value corresponding to each actual value. The response distances are shown in Figure 3 and the response angles in Figure 4. Each panel shows two contrasted conditions. The upper panels show the effects of number of starting points within the trans and no-trans conditions (upper left and upper right panels, respectively); the lower left panel shows the rotation condition and its large-trans control, and the lower right panel shows the location-match condition and the matched no-trans/ 1 condition (duplicated from the upper right panel). For each condition, the best-fitting linear function relating observed to actual distance or angle is indicated. The $r$ values for these linear fits ranged from .92 to .99 . The diagonal indicates accurate responding, with values below the diagonal being underresponding and those above it overresponding.

Some analyses use signed and absolute error in response distance or angle. The signed error was defined as observed response value minus actual value. The absolute error was the absolute value of the signed error.

\section{Learning and Individual-Difference Analyses}

Initially, each condition was examined for learning effects and for individual differences in mental rotation ability. To examine learning, signed error and absolute error in response distance and angle were first regressed on the actual response, separately for each subject. The correlation was then determined between the residuals of the regression, which represent errors that cannot be predicted by the path parameters, and the order in which the pathways occurred for that subject. To examine the effects of spatial ability, correlations were measured between errors and the mental rotation score. Both the learning and the mental rotation correlations were uniformly low, and these analyses will therefore not be discussed further.

\section{Principal Analyses}

The principal analyses isolate subgroups of the overall design to make particular comparisons. For these purposes, analyses of variance (ANOVAs) were conducted on the signed errors in the observed response distance and observed response angle. The ANOVAs included path as a factor (with 27 levels) and other factors defined by the groups that were included in the analysis. In reporting these ANOVAs, all significant effects are stated, as well as nonsignificant effects of particular interest.

Table 1 indicates the magnitude of the mean signed error in each condition, averaged across pathways, and the standard deviation of that mean (computed over subjects). To the extent that the errors tended to be signed similarly across pathways, as can be seen in Figures 3 and 4, the average signed error represents an overall tendency rather than an artifactually low value resulting from cancellation of errors with different signs.

Tables 2 and 3 report correlations between all pairs of conditions with respect to signed error in observed response distance and observed response angle. It is noteworthy that the distance errors tended to be highly correlated across the various conditions, but the angle errors tended to be uncorrelated. 

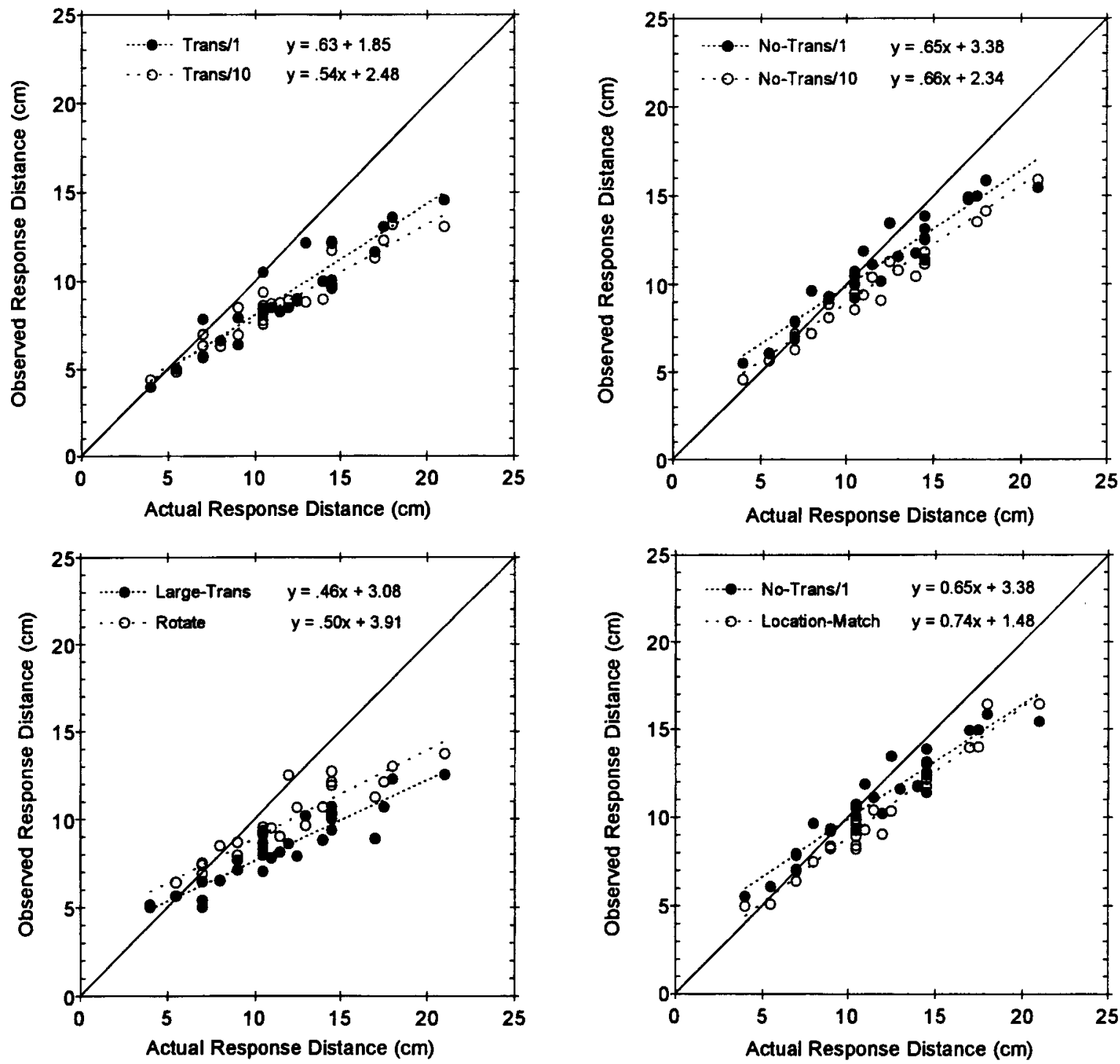

Figure 3. Observed response distance corresponding to each value of actual response distance and best-fitting linear function for the experimental conditions. Each panel contrasts two conditions; the no-trans/1 condition is duplicated in the upper and lower right panels. The diagonal in each panel shows correct performance.

\section{Effects of Translation and}

\section{Number of Starting Points}

To examine the effects of translation and number of starting points, the ANOVAs on signed errors in observed response distance and observed response angle involved four groups: trans $/ 1$, trans $/ 10$, no-trans $/ 1$, and no-trans $/ 10$. The factors for each $2 \times 2 \times 27$ ANOVA were translation (present or absent), number of starting points ( 1 or 10$)$, and path. To preview the most important results, the effect of number of starting points ( 1 vs. 10) was not significant for either measure, and the effect of translation was significant only for distance error.

Figure 3 (upper panels) shows the observed response distance as a function of the actual value for the trans and no-trans conditions, and for each number of starting points. As can be seen, subjects tended to underrespond, increas-

Table 1

Mean Signed Error and Standard Deviation, Computed Across Subjects, Within Each Condition*

\begin{tabular}{|c|c|c|c|c|c|c|c|c|c|c|c|c|c|c|}
\hline & \multicolumn{2}{|c|}{ Trans/1 } & \multicolumn{2}{|c|}{ Trans $/ 10$} & \multicolumn{2}{|c|}{ No-Trans/1 } & \multicolumn{2}{|c|}{ No-Trans/10 } & \multicolumn{2}{|c|}{ Rotation } & \multicolumn{2}{|c|}{ Large-Trans } & \multicolumn{2}{|c|}{ Location-Match } \\
\hline & $M$ & $S D$ & $M$ & $S D$ & $M$ & $S D$ & $M$ & $S D$ & $M$ & $S D$ & $M$ & $S D$ & $M$ & $S D$ \\
\hline & $\begin{array}{r}-2.5 \\
176\end{array}$ & $\begin{array}{r}1.2 \\
23.3\end{array}$ & $\begin{array}{r}-2.9 \\
3.7\end{array}$ & $\begin{array}{r}2.2 \\
127\end{array}$ & $\begin{array}{l}-0.7 \\
-40\end{array}$ & 1.8 & & 85 & $\begin{array}{r}-1.9 \\
26 ?\end{array}$ & 1.2 & & 0.6 & -1.8 & \\
\hline
\end{tabular}

*The average actual response value is a distance of $11.6 \mathrm{~cm}$ and an angle of $45.6^{\circ}$. 

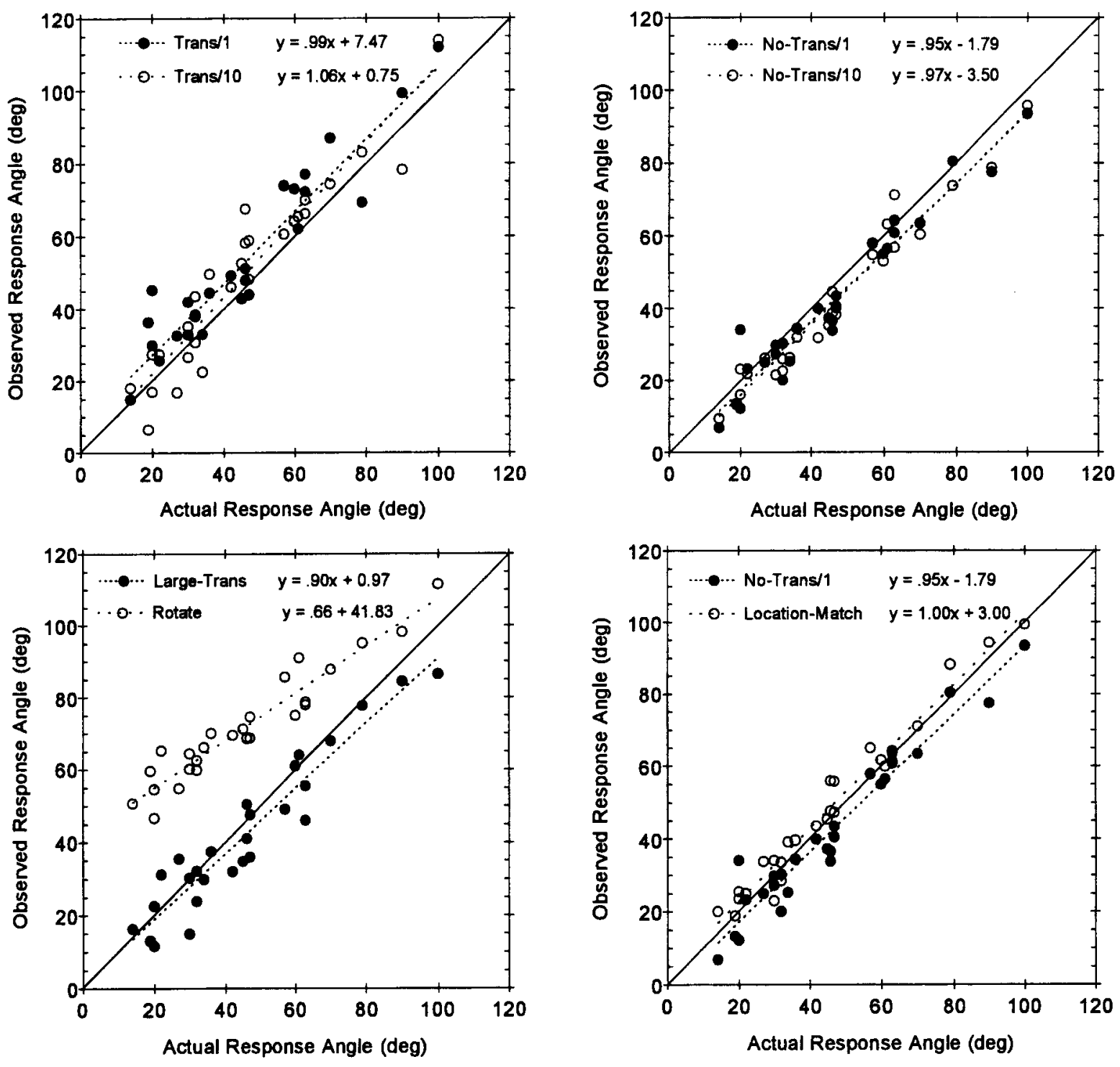

Figure 4. Observed response angle corresponding to each value of actual response angle and best-fitting linear function for the experimental conditions. Each panel contrasts two conditions; the no-trans/1 condition is duplicated in the upper and lower right panels. The diagonal in each panel shows correct performance.

ingly so as the actual response distance increased, and this error tendency was greater following translation. Accordingly, the ANOVA on signed error in observed response distance revealed an effect of translation $[F(1,36)=7.29$, $p<.05]$ and path $[F(26,936)=24.67, p<.001]$. There was no effect of the number of starting points $(p>.125)$.

Figure 4 (upper panels) shows the angle response as a function of the actual value for the trans and no-trans conditions, with 1 and 10 starting points. The ANOVA showed no significant effects, although the path effect was marginal $[F(26,936)=1.49, p=.05]$. This effect largely reflects unsystematic variation across pathways, and the errors were not correlated across conditions. Like the distance errors, the angle errors showed no effect of number of starting points $(p>.50)$.

\section{Effects of Instructions to Match Location}

This analysis compared the no-trans/1 and locationmatch conditions, which differed only in that the first group was told to complete the triangle and the second group was told that the origin was in a constant location in space and that they should try to return to it on each trial despite having traveled over the pathway. The relevant data are in Figures 3 and 4, bottom right. The ANOVA was conducted on instructions and path. The two groups were very similar, and neither the distance ANOVA nor the angle 
Table 2

Correlations Between Conditions Across Pathways: Signed Distance Error*

\begin{tabular}{lrrrrrrr}
\hline & Trans/1 & Trans/10 & No-Trans/1 & No-Trans/10 & Rotation & Large-Trans & Location-Match \\
\hline Trans/1 & 1.00 & & & & & & \\
Trans/10 & .81 & 1.00 & & & & & \\
No-trans/1 & .79 & .84 & 1.00 & & & & \\
No-trans $/ 10$ & .89 & .90 & .86 & 1.00 & & & \\
Rotation & .76 & .81 & .78 & .89 & 1.00 & & \\
Large-trans & .91 & .86 & .80 & .93 & .91 & 1.00 & .88 \\
Location-match & .85 & .81 & .83 & .86 & .75 & .00 \\
\hline
\end{tabular}

${ }^{*}$ In all cases, $d f=25$, critical value at .05 , two-tailed, $=.38$.

Table 3

Correlations Between Conditions Across Pathways: Signed Angle Error*

\begin{tabular}{|c|c|c|c|c|c|c|c|}
\hline & Trans/ 1 & Trans/10 & No-Trans/1 & No-Trans/10 & Rotation & Large-Trans & Location-Match \\
\hline Trans/1 & 1,00 & & & & & & \\
\hline Trans/10 & .20 & 1.00 & & & & & \\
\hline No-trans /1 & .23 & -.17 & 1.00 & & & & \\
\hline No-trans $/ 10$ & -.04 & -.03 & .61 & 1.00 & & & \\
\hline Rotation & .13 & -.14 & .24 & .20 & 1.00 & & \\
\hline Large-trans & -.03 & .06 & .10 & .29 & .40 & 1.00 & \\
\hline Location-match & -.18 & .01 & .05 & .12 & .05 & .39 & 1.00 \\
\hline
\end{tabular}

*In all cases, $d f=25$, critical value at .05 , two-tailed, $=.38$.

ANOVA showed an effect of instruction. Both ANOVAs revealed only an effect of path - for signed distance errors $[F(26,364)=8.83, p<.001]$ and for signed angle errors $[F(26,364)=1.59, p<.05]$.

\section{Effects of Rotation Versus Translation}

These ANOVAs compared the no-trans/10, large-trans, and rotation conditions, all of which used 10 starting points, in a three-level factor, displacement type. The 27 paths constituted a second factor. The lower panels of Figures 3 (distance) and 4 (angle) show the responses in the rotation and large-trans conditions, as a function of the actual value, across the 27 paths. The results for the notrans/10 condition are shown in the upper right panels of the corresponding figures. To preview the results, the rotation substantially affected angle errors but had no effect on distance errors; conversely, the effects of the large $(9.5-\mathrm{cm})$ translation, like those of the smaller $(4-\mathrm{cm})$ translations described above, were confined to the distance errors.

The ANOVA on distance error produced an effect of path $[F(26,546)=25.26, p<.001]$, again reflecting an increasing underresponse tendency as the actual response distance value increased. Although the overall displacement effect (rotation vs. large-trans vs. no-trans/10) was not significant, the path $\times$ displacement effect was $[F(52,546)=$ $1.76, p<.001]$, indicating that a displacement effect emerged with greater actual response distances (where errors were greater). Follow-up ANOVAs were conducted to compare the displacement conditions pairwise. There was not a significant difference in signed distance error between the no-trans/ 10 and rotation conditions (see Table 1). The comparison between the large-trans condition and each of the other two showed that the translation produced an increase in the underresponse error tendency. In the analysis comparing large-trans with no-trans $/ 10$, the trans- lation effect was particularly evident at the longer paths, producing a significant path $\times$ displacement interaction $[F(26,364)=1.97, p<.01]$. In the analysis comparing large-trans and rotation, there was a significant main effect of displacement $[F(1,14)=5.00, p<.05]$.

The ANOVA on angle error with factors of path and displacement (rotation vs. large-trans vs. no-trans/10) showed an effect of path $[F(26,546)=2.48, p<.001]$ and displacement $[F(2,21)=21.91, p<.001]$. The displacement effect arose because of the obviously larger error for the rotation condition (see Figure 4, bottom left). Followup ANOVAs were used to compare the displacement conditions pairwise with respect to angle error. The large-trans and no-trans $/ 10$ conditions did not differ significantly. The error in the rotation condition was greater than in the largetrans condition $[F(1,14)=27.81, p<.001]$ or the no-trans/ 10 condition $[F(1,14)=34.96, p<.001]$. In the rotation versus no-trans $/ 10$ comparison, the path $\times$ displacement effect was also significant $[F(26,364)=1.98, p<.01]$, reflecting the tendency for errors in the rotation condition to be greater at smaller values of actual response angle.

\section{Fitting the Encoding-Error Model}

The fit of the encoding-error model to the data is next described. To fit the model, conditions were pooled that showed no significant differences. One data set consisted of the conditions with no translation (no-trans/1, no-trans/10, and location-match). A second data set pooled conditions with translation (trans/1, trans/10, and large-trans-note that ANOVAs comparing the trans/ 10 and large-trans conditions, with translations of 4 and $9.5 \mathrm{~cm}$, respectively, showed no effects involving magnitude of translation). The third data set came from the rotation condition.

Because subjects in the conditions with and without displacements were treated identically up to the point of the 

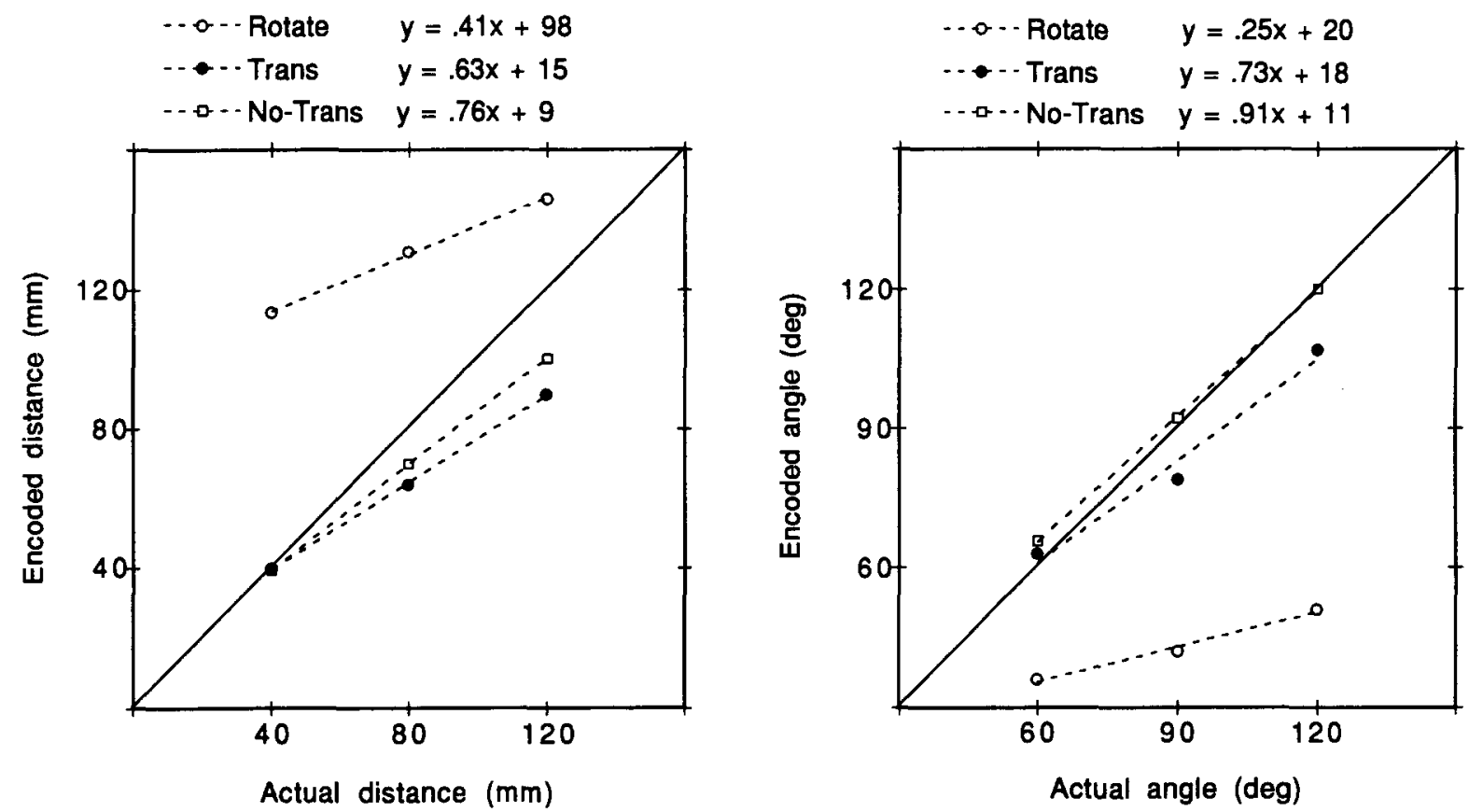

Figure 5. Encoding functions for distance and angle, derived from the encoding-error model for each pooled data set, showing the encoded value corresponding to each stimulus parameter (distance of Leg 1 or 2; Angle 1 value). The best-fitting linear function is also shown.

response, the initial processes used to encode the path parameters should be identical. Differences in the parameters fit by the model would therefore represent changes in the internal representation of the path that were attributable to the displacement-that is, changes that occurred subsequent to initial encoding of the outbound path and that were engendered by rotating or translating the finger.

To fit the model, we first estimated the six parameters corresponding to the encoded value for each actual value of leg length $(4,8$, and $12 \mathrm{~cm})$ and internal angle $\left(60^{\circ}, 90^{\circ}\right.$, and $120^{\circ}$ ) in the triangle. As was found for the analysis of Fujita et al. (1993), the parameters fell on a linear function (in all cases, $r \geq .99$ ), allowing the parameters of the model to be reduced from six (the encoded value corresponding to each stimulus value) to four (the slope and intercept of the encoding functions). The parameter estimates for each data set are shown in Figure 5. Although the form of the encoding functions was linear, as was found previously, the functions did not generally exhibit highest accuracy at the mean of stimulus values. Only the angle encoding function fit to the no-translation data set met the diagonal near the mean of actual stimulus values $\left(90^{\circ}\right)$; however, the entire function lay essentially along the diagonal rather than crossing it there.

Most importantly, the encoding functions for the rotation condition were much further from the diagonal (which indicates correct encoding) than the encoding functions for the trans and no-trans conditions. As a consequence of rotation, subjects appear to have represented leg lengths as larger than they actually were and to have represented an- gles as smaller than they actually were. They also considerably compressed the range of encoded values relative to actual ones. The model indicates that after rotation, leg lengths were represented as approximately $12-14 \mathrm{~cm}$, and most angles were represented as approximately $35^{\circ}$ to $50^{\circ}$. The inaccuracies in the encoding functions for the other conditions are far less pronounced. The no-translation functions were closer to the diagonal than the translation functions, as is consistent with the higher error under translation.

The goodness-of-fit of the model to the data is shown in Figures 6 (trans and no-trans data) and 7 (rotation data). Each panel shows the predicted and observed response (for distance or angle, as indicated) as a function of the actual response value. It is important to consider not only whether the model predicts the general trends in the data (e.g., that the observed response distance increases as the actual response distance increases), but also whether it predicts the precise response values obtained. The goodness of fit to individual data points can be assessed by the match between the predicted and observed signed error in the response distance or angle; this measure is not inflated by a gross prediction of increasing response values with increasing actual values. The correlation between predicted and observed error, and the slope of the function relating them, are given for each data set in Table 4. The higher the correlation and the closer the slope to 1.0 , the better the model predicts the data.

Within the trans and no-trans data sets, the distance errors were fit much better than the angles. At the outset, it was clear that the model would have difficulty in predict- 

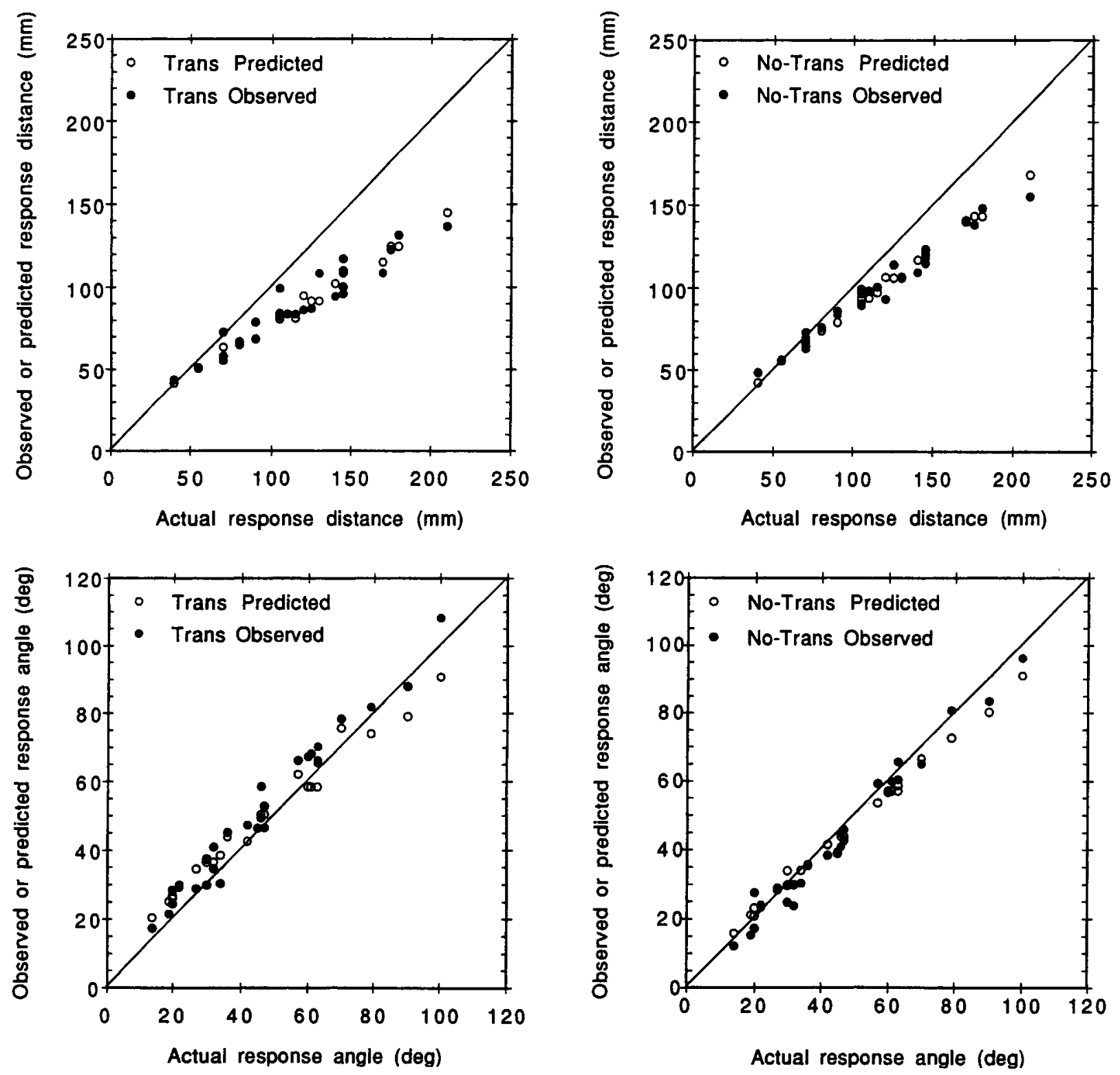

Figure 6. Observed (filled circles) and predicted (open circles) response distance (upper panels) or angle (lower panels) as a function of actual response distance or angle. The left and right panels show the model predictions for the pooled translate and no-translate conditions, respectively.

ing the observed angle errors in those data sets, because (1) the angle responses clustered around the diagonal of the response versus actual function rather than showing systematic departures from accuracy (see Figure 4), and (2) the error pattern was not correlated across the various conditions that entered the pooled data set (see Table 3). In other words, the observed angle errors in the trans and no-trans data sets appear to be more attributable to noise than to systematic sources, making them difficult to predict. ${ }^{2}$ As is shown in Figure 6, what the model predicted for angle responses was a tendency for increased underresponding in the observed response angle, the greater the actual response angle. This is the same systematic tendency as was found for the distance responses, but it was not observed in the angle response data, in contrast to the model's prediction.

Unlike the no-trans and trans conditions, the rotation condition yielded both distance and angle errors that were systematically related to path parameters. The model accounted for $81 \%$ of the variance in distance responses. Its fit to the angle responses was somewhat worse $(66 \%$ of variance), particularly because it failed to predict the tendency to overrespond at the greater values of actual response angle (see Figure 7). As discussed below, that tendency may reflect processes outside the scope of the model- 

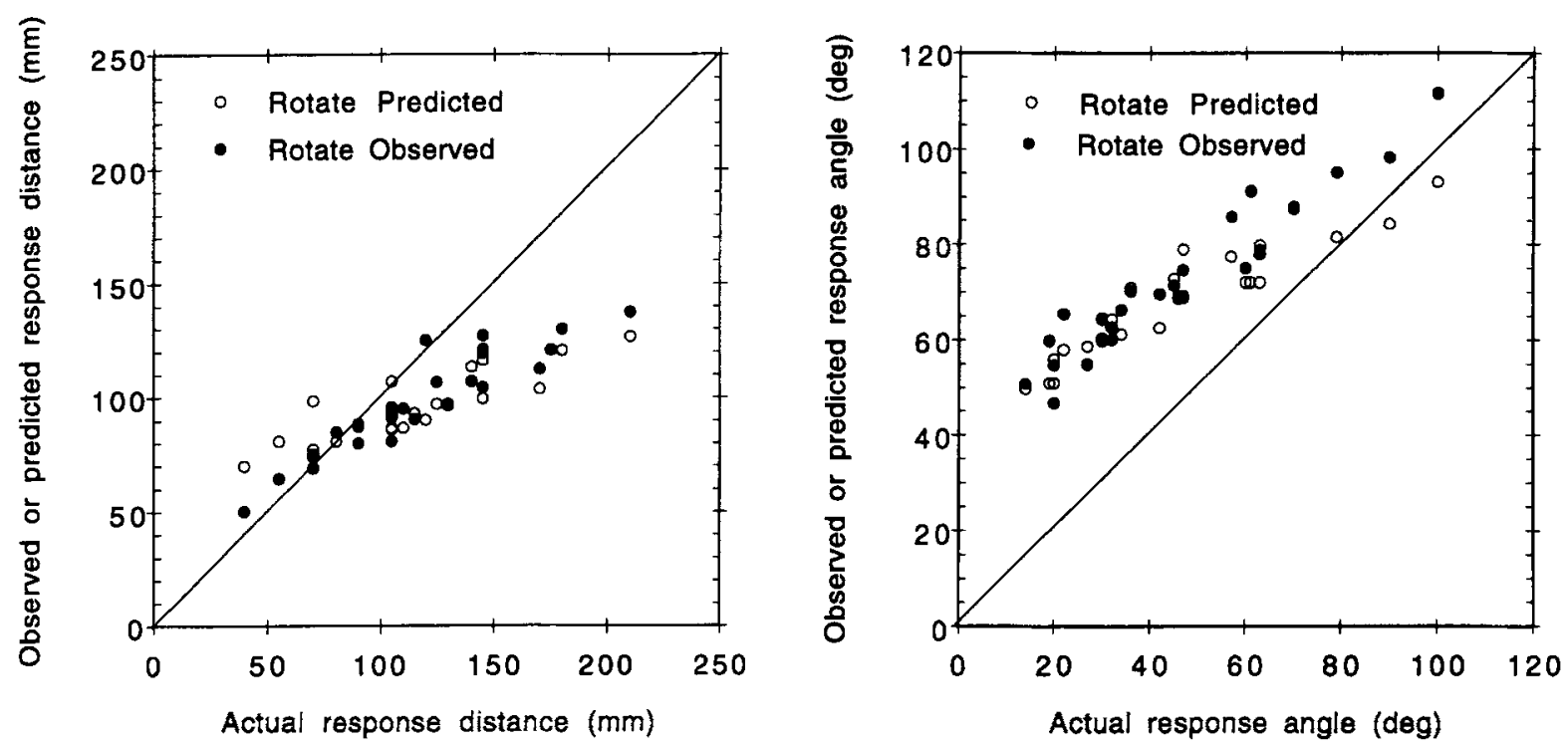

Figure 7. Observed (filled circles) and predicted (open circles) response distance (left panel) or angle (right panel) as a function of actual response distance or angle in the rotation condition.

specifically, a bias to counteract the rotation by orienting the response back toward the original starting point.

\section{DISCUSSION}

The present study addressed three general issues. One was whether path completion in space on the scale of a tabletop (manipulatory space) could be accounted for by mechanisms similar to those used to model completion of a walked path in larger scale (ambulatory) space. The particular mechanisms of interest were those described by the encoding-error model. On the whole, the data indicate that the encoding-error model does not provide a general account of path completion in manipulatory space. In contrast to predictions of the model, people appear to be quite accurate when determining the direction from the end of the outbound path to the origin, while at the same time they are subject to systematic error when determining the distance between those points.

A second issue was whether imagined translation and rotation before responding would have effects of different magnitude, as has been found with locomotor tasks. As in the experiments in ambulatory space, the present study indicates that rotation had considerably greater impact. Moreover, the effect was exclusive to the angle component of the response, and not the distance component. In contrast, translation affected the distance component and not the response angle.

The third issue concerned the nature of the spatial representations and processes used to compute distance and angle responses. The data from the no-displacement and translation conditions are consistent with the use of a reference system that anchors the path in space. Results of the rotation condition are more consistent with an object- centered representation, in which case the parameters fit to the data by the encoding-error model provide a description of the consequences of rotation for that representation.

Subsequent sections of the discussion develop and document these conclusions. The discussion considers not only the encoding-error model, but also other phenomena in haptic space perception that might be proposed to account for the results.

\section{Similarity of Path Completion Responses (Angle and Distance) in Manipulatory and Ambulatory Space}

The introduction raised the point that exploration without vision in manipulatory and ambulatory space differs not only in the scale that is explored, but also in that the body provides a fixed frame of reference during manipulatory exploration but not during locomotion. Although scale could potentially influence processing in its own right (see, e.g., Acredolo, 1981), this paper emphasizes the potential contribution of the fixed reference frame. The

Table 4

Fit of the Model to the Distance and Angle Responses in the Three Data Sets*

\begin{tabular}{|c|c|c|c|c|}
\hline \multirow[b]{2}{*}{ Data Set } & \multicolumn{2}{|c|}{$\begin{array}{l}\text { Correlation } \\
\text { Between Observed } \\
\text { and Predicted Error }\end{array}$} & \multicolumn{2}{|c|}{$\begin{array}{l}\text { Slope of Function } \\
\text { Relating Observed } \\
\text { and Predicted Error }\end{array}$} \\
\hline & Distance & Angle & Distance & Angle \\
\hline No-trans & $.88 \dagger$ & .25 & .61 & .26 \\
\hline Trans & $.92 \dagger$ & .07 & .79 & .10 \\
\hline Rotation & $.90^{\dagger}$ & $.81 \dagger$ & 1.18 & 1.14 \\
\hline
\end{tabular}

*As indicated by (1) the correlation between predicted error and actual error and (2) the slope of the function relating predicted error to actual error. The correlation and siope are computed across the 27 paths. ${ }^{\dagger} p<$ .01 . 
data suggest that differential availability of an external frame of reference produces differences between haptic exploration and locomotion in path-completion processes.

A comparison of the present data to findings from the triangle-completion task in ambulatory space indicates that there were similar trends with respect to the distance responses, but not angle responses. More specifically, the observed response distance showed a highly regular pattern in relation to the actual response distance; there was increased underresponding as the value of the actual response increased. The underresponding to longer values of actual response distance produced compression of the range of observed response distances relative to the range of actual values. This is reminiscent of effects found in ambulatory triangle completion (Klatzky et al., in press; Loomis et al., 1993), although those studies showed not only underresponding to long distances, as was found here, but also overresponding to short distances, which was not present in these data. The regular pattern of underresponding to longer values of actual response distance was found in every condition in the study, and as a result, distance errors tended to be highly intercorrelated.

In contrast to the systematic nature of the distance responses, the angle responses in all but the rotation condition took the form of unsystematic under- and overresponding around the actual response angle. This pattern is quite different from the data obtained by Loomis et al. (1993) for ambulatory triangle completion. Moreover, the deviations from the correct angular value did not correlate across conditions. The contrast between the systematic error in distance responses and the seemingly stochastic variations in angle responses (in the absence of rotation before responding) suggests that the two parameters are computed by different processes.

Evidence for a dissociation between haptic representation of angle and distance has also been found by Fasse, Hogan, Kay, and Mussa-Ivaldi (1994). They had subjects trace the boundaries of virtual planar shapes, using a robotic manipulandum with force reflection, and then judge the relative length of two sides or the relative size of angles. Significant perceptual distortions of length and angle (both around 30\%) were obtained; however, the angle distortion could not be predicted from the distortion of component leg lengths.

Another indication of a dissociation between angle and distance representations in manipulatory space can be found in the present data, in terms of the selective effects of the preresponse displacements (rotation vs. translation). The next section describes those effects.

\section{Effects of Preresponse Rotation Versus Translation on Distance and Angle Responses}

As noted, the displacements were selective in their effects. Translational displacement exclusively affected distance error, and rotational displacement exclusively affected angle error. Moreover, the magnitude of the rotation effect was considerably greater than that of the translation effect. Depending on the conditions that were compared (see Table 1), translation caused up to a four-fold increase in the average distance error. Essentially, the subjects became more conservative in their distance responses after translation; the entire function relating observed to actual response values shifted downard, indicating shorter distance responses across all pathways. However, the effect of the translation on angle responses was inconsistent in direction and not significant in magnitude. Conversely, the rotation displacement increased the average magnitude of angle error ten-fold, without affecting distance error.

The difference between rotation and translation is supported by the results of fitting the encoding-error model. When the model was fit to the data from the translation and no-translation conditions, it indicated that the internal representation was changed only modestly by the linear displacement. Although one should be wary of interpreting the functions given the fits of the model, this is consistent with the relatively small effects of translation on the observed errors. In contrast, when fit to the rotation data, the model produced a very different encoding function, which indicated considerable distortion in the internal representation of the path.

\section{Representation and Processes Underlying Performance With No Displacement or a Translation Displacement}

The encoding-error model proposes one account of the underlying basis for responses in the task, but there is little support for its assumptions under conditions with no displacement or translational displacement before responding. The dissimilarity between the level of systematic error in angle and distance responses is inconsistent with the model, which assumes that both types of errors arise from a common source - misrepresenting the leg lengths and turn in the outbound path. Moreover, the model made specific predictions about angle errors, which were not confirmed. What it predicted for angles was the same tendency as was actually found for distances - that is, increased underresponding with greater actual stimulus values. But that pattern was not found in the observed response angles.

If the encoding-error model does not apply to the present data from the no-trans and trans conditions, how do subjects compute the distance and angle responses? Given the equivalence of location-match and triangle-completion instructions, one might be tempted to assume that in either case, subjects were attempting to ignore the triangle and code the location of the origin relative to the body. With such a strategy, however, one would expect an effect of the variability of that location (i.e., an effect of number of starting points) and a tendency to improve over trials when the location was held constant; neither effect was observed. The most telling evidence against coding only the origin with respect to the body is found in the negligible effects of translating its location before responding. Even the large translation had no effect on response angle errors and only a small effect on response distance errors. If subjects were relying on a fixed location relative to the body, the data would seem to dictate that they were able to trans- 
late that location while incurring virtually no error. That seems unlikely, given that a translation would change both the distance and the angle of the origin from the subject, and the changes would increase with the magnitude of the translation.

Thus far two hypotheses for the no-displacement and translation conditions have received little support from the present data: It appears that subjects did not encode parameters of the path independently of its spatial position (i.e., in an object-centered frame), as proposed by the encoding-error model, nor did they simply attempt to position the origin relative to the body and ignore the outbound path. The third alternative, and the one most consistent with the present data, is that subjects represented the triangular path configuration within a reference system anchored in space external to it. The natural anchors for such a system would be the body and/or fixed, haptically accessible features such as the table edge. The pathway coordinates could be represented egocentrically (i.e., relative to the body) or allocentrically (in terms of referents outside the body), but in either case, the pathway would be localized in space rather than within an objectcentered representation.

In a non-object-centered representation, the response angle can be represented as a bearing from the end of the second leg to the origin, relative to some reference axis (e.g., the body's saggital plane). The response distance is independent of the response angle in the sense that the triangle can be proportionally rescaled without changing the angles. In such a representation, neither the response distance nor the response angle (bearing) changes with translation, which is consistent with the finding that translation had little or no effect on levels of error. However, if the response bearing is computed relative to a non-objectcentered reference axis, it does change with rotation, which is discussed next.

\section{Representation and Processes Underlying Performance With Rotation}

Processing subsequent to a rotation appears to be quite different from processing in the other conditions. The degree of fit of the encoding-error model suggests that the model's assumption of object-centered processing may hold for this condition. If applied to the rotation data, the model must be invoked after the initial encoding of the path, since the rotation occurred just before the response. Thus, rotation subjects can be assumed to begin the task just as in the other conditions, by representing the triangle within a reference system external to the path. However, the model stipulates that they ultimately attempted to respond on the basis of an object-centered representation; that is, they used encoded values of Leg 1 , Leg 2, and Angle 1 to compute the responses. The encoding functions derived from the rotation condition then describe rotationinduced degradation in the internal representation of the path parameters. The model indicates that rotation caused subjects to represent all the paths almost uniformly-as having leg lengths of about $12 \mathrm{~cm}$ and angles of about $45^{\circ}$.
At the same time, there is some evidence that subjects in the rotation condition were influenced by memory for the initial location of the origin in external spatial coordinates. Consider that the angle error after rotation took the form of overresponding (i.e., producing an inner angle in the triangle that was too wide). This trend would result if subjects tended to direct their finger rightward, back toward the original location of the origin, after the clockwise rotation. ${ }^{3}$ The bias toward rightward responuing was particularly evident when the actual response angle was small, but this can be interpreted somewhat differently: Because pathways with smaller values of response angle tended to have larger values of response distance (the correlation across paths between the actual response distance and angle is $r=-.30$ ), this means that the bias toward rightward responding increased, the further the finger was from the correct response location (i.e., the displaced origin) at the time of the response. ${ }^{4}$ This bias was a source of systematic error that was not predicted by the encodingerror model.

Ultimately, the data suggest that subjects in the rotation condition used a combination of processes after the rotation-responding in part on the basis of a degraded, objectcentered representation of the outbound path, as described by the model, with an additional bias based on memory for the location of the origin within an externalized reference frame.

\section{Contrast Between Translational and Rotational Displacements}

The finding that rotation affects angular error more than translation, when responses are made in manipulatory space, parallels the phenomenon found in ambulatory space: Updating angular position relative to objects in space is known to be easier when people imagine themselves translating than when they imagine themselves rotating (Rieser, 1989). In attempting to account for this phenomenon, Rieser noted that translation of the body does not change the direction (or bearing) from one object to another in the space. He proposed that people have access to object-to-object directions after translation, and that they respond by indicating the known direction. Rotations, in contrast, do change the directions from one object to another.

Similarly, in the present situation, rotation, but not translation, alters the bearing from the end of Leg 2 to the origin, which corresponds to the actual response angle. This change in the bearing occurs if a reference system external to the path is used; if an object-centered representation is used, in contrast, the bearing does not change. The ease with which subjects handled translations, but not rotations, supports the idea that they used a non-object-centered, externalized reference system (although presumably defaulting to an object-centered one after rotation).

The present results are consistent with previous findings of Millar, who has conducted a number of studies investigating the means by which blind and sighted individuals code spatial locations within manipulatory space 
(summarized in Millar, 1994; see also Ballesteros, Millar, \& Reales, 1998). In particular, she has attempted to differentiate among body-centered, non-body-centered, and movement-based spatial reference systems. Her findings suggest that in the absence of vision, a body-centered reference (particularly the sagittal axis) is preferred, but movement coding and externally based systems will be called in when the use of the body is made difficult. One such circumstance is when rotation of the spatial layout or the body is required between original encoding and response (Millar, 1976). Her work further suggests that the alternative to body-centered coding adopted by blind and sighted individuals may differ, with the blind favoring movement-based coding and the sighted favoring use of a non-body-centered spatial reference frame. In accord with these proposals, data from the sighted subjects in the present no-translation and translation conditions are consistent with a body-defined reference frame. (An important point, however, is that the entire pathway is represented within that frame, not just the position of the origin.) And again as Millar has suggested, mental rotation of the pathway apparently leads to an alternative spatial code. The present data further indicate that the code subsequent to rotation is object centered and vulnerable to errors described by the encoding-error model.

Klatzky, Loomis, Beall, Chance, and Golledge (1998) have suggested that the difficulty in dealing with imagined rotations of the body arises because egocentric spatial representations, which convey the bearings of objects relative to an individual's heading in space, are normally updated by inputs from vestibular cues, and under imagined rotation of the body, those cues are absent. In the present task, the subjects were asked to imagine a triangle rotating, not their bodies. Vestibular cues would not be present if the triangle physically rotated, and hence could not be said to be absent when the triangle imaginally rotated. Nevertheless, it appears that subjects had difficulty with updating the position of the origin when they imagined the triangle rotating relative to their stationary bodies, as they would if they imagined themselves rotating without vestibular input.

\section{Relation of the Findings to Other Phenomena in Haptic Distance Perception}

It has been suggested that the present findings are best accounted for by a mechanism that uses a spatially anchored reference system as a basis for coding the location of a haptically explored path. This section considers the relation of these findings to other mechanisms that have been proposed to account for haptic perception of manipulatory space.

First, the present study provides no evidence for use of a movement-based heuristic to estimate distance, like that described by Lederman et al. (1987). In that study, subjects tended to overestimate the length of the third leg of a triangle; this tendency was greater the longer the indirect path. However, underresponding was the norm in the pres- ent study, despite the use of an indirect outbound path. Another prediction of the movement-based heuristic is that the mean observed response distance will be correlated with the total length of the first two legs. When that correlation was computed across pathways for each of the present conditions, the correlations tended to be high.69 to .80 . However, before taking the correlation as evidence for use of the movement heuristic, one must consider that the total length of the first two legs would be highly correlated with the actual response distance even if the subjects performed without error $(r=.81)$. In other words, the predicted correlation could be confirmed even if subjects responded with complete accuracy, not using the heuristic at all. The more telling test of the heuristic is to compute the correlation with actual response distance partialed out. The correlation between the total of the first two legs and the observed response distance, with actual response distance removed, ranged from -.25 to .18 across conditions. This offers no support for use of the heuristic. There are procedural differences between the present study and that of Lederman et al. that might have discouraged use of a movement-based heuristic. Two differences that might have affected subjects' processing was the use of a motor response here rather than symbolic estimation, and the fact that pathway distance and response distance were correlated here rather than decoupled.

Two other phenomena of haptic perception described in the introduction are the oblique effect and the radial/ tangential effect. The present studies were not intended to assess these effects directly, but there is little evidence that they account for the data. First, the lack of correlation in the angle errors across conditions suggests that systematic angular anisotropies such as the oblique effect were not a basis for the results. Conversely, the fact that distance responses were highly correlated across conditions, including high correlations between conditions where the starting point was varied and those where it was held constant, suggests that variations in the radial versus tangential components of the movements did not contribute strongly to the data either.

\section{Summary and Conclusion}

Path completion in manipulatory space appears to capitalize on the possibility of using a reference system that can be localized with respect to the body. Such a system is not available when subjects repeatedly complete paths by locomotion-that is, in ambulatory space. The reference system appears to provide highly accurate representation of the bearings between points on a haptically explored path and less accurate representation of interpoint distances. Morever, lateral translations of the explored configuration are relatively easy to accommodate, whereas rotations induce considerable error. There is some indication that rotation produced a shift in strategy away from a body-centered reference system and toward object-centered processes used to complete paths in ambulatory space, as described by the encoding-error model. 


\section{REFERENCES}

ACREDOLO, L. P. (1981). Small- and large-scale spatial concepts in infancy and childhood. In L. S. Liben, A. H. Patterson, \& N. Newcombe (Eds.), Spatial representation and behavior across the life span: Theory and application (pp. 63-81). New York: Academic Press.

ApPElle, S., \& Countryman, M. (1986). Eliminating the haptic oblique effect: Influence of scanning incongruity and prior knowledge of the standards. Perception, 15, 365-369.

Ballesteros, S., Millar, S., \& Reales, J. M. (1998). Symmetry in haptic and in visual shape perception. Perception \& Psychophysics, 60, 389-404.

ChEnG, M. F. H. (1968). Tactile-kinesthetic perception of length. American Journal of Psychology, 81, 74-82.

Etienne, A. S., Maurer, R., \& SÉGuinot, V. (1996). Path integration in mammals and its interaction with visual landmarks. Journal of Experimental Biology, 199, 201-209.

Fasse, E. D., Hogan, N., Kay, B. A., \& Mussa-Ivaldi, F. A. (1994). Haptic interaction with virtual objects: Spatial perception and motor control. Unpublished manuscript.

Fuitta, N., Klatzky, R., Loomis, J. M., \& Golledge, R. G. (1993). The encoding-error model of pathway completion without vision. Geographical Analysis, 25, 295-314.

Gallistel, C. R. (1990). The organization of learning. Cambridge, MA: MIT Press.

Gentaz, E., \& Hatwell, Y. (1995). The haptic "oblique effect" in children's and adults' perception of orientation. Perception, 24, 631-646.

Klatzky, R. L., Beall, A. C., Loomis, J. M., Golledge, R. G., \& PhILBECK, J. W. (in press). Human navigation ability: Tests of the encoding-error model of path integration. Spatial Cognition \& Computation.

Klatzky, R. L., Loomis, J. M., Beall, A. C., Chance, S. S., \& GolLEDGE, R. G. (1998). Spatial updating of self-position and orientation during real, imagined, and virtual locomotion. Psychological Science, 9, 293-298.

KlatzKy, R. L., Loomis, J. M., \& GolledGe, R. G. (1997). Encoding spatial representations through nonvisually guided locomotion: Tests of human path integration. In D. L. Medin (Ed.), The psychology of learning and motivation (Vol. 37, pp. 41-84). San Diego: Academic Press.

Lederman, S. J., Klatzky, R. L., Collins, R., \& Wardell, J. (1987). Exploring environments by hand and foot: Time-based heuristics for encoding distance in movement space. Journal of Experimental Psychology: Human Learning, Memory, \& Cognition, 13, 606-614.

Loomis, J. M., Klatzky, R. L., Golledge, R. G., Cicinelli, J. G., PeLLEGRINO, J. W., \& FRY, P. (1993). Nonvisual navigation by blind and sighted: Assessment of path integration ability. Journal of Experimental Psychology: General, 122, 73-91.

Marchetti, F. M., \& Lederman, S. J. (1983). The haptic radialtangential effect: Two tests of Wong's "moments-of-inertia" hypothesis. Bulletin of the Psychonomic Society, 21, 43-46.
MARR, D. (1982). Vision. San Francisco; Freeman.

MAURER, R., \& SÉGuinot, V. (1995). What is modelling for? A critical review of the models of path integration. Journal of Theoretical Biology, 175, 457-475.

MILLAR, S. (1976). Spatial representation by blind and sighted children. Journal of Experimental Child Psychology, 21, 460-479.

MILLAR, S. (1994). Understanding and representing space: Theory and evidence from studies with blind and sighted children. New York: Oxford University Press.

RIESER, J. J. (1989). Access to knowledge of spatial structure at novel points of observation. Journal of Experimental Psychology: Learning, Memory, \& Cognition, 15, 1157-1165.

RIESER, J. J., \& FrYMIRE, M. (1995, November). Locomotion with vision is coupled with knowledge of real and imagined surroundings. Paper presented at the thirty-sixth annual meeting of the Psychonomic Society, Los Angeles.

\section{NOTES}

1. Under other circumstances, however, subjects performing path completion without vision may make use of external features. Rieser and Frymire (1995) reported that having subjects imagine the path within a larger spatial context facilitated performance. In this case, subjects could imaginally update their position relative to contextual landmarks while walking on the path and could compute responses on the basis of those landmarks.

2 . We conducted a variation on the model to see if a better fit could be obtained without considering the angle responses, given their unsystematic pattern. The measure of model error was based only on the difference between predicted and observed distance responses. The revised model did improve the predicted distance responses (the functions relating predicted error to observed error had $r$ values of .96 and .94 and slopes of .92 and .89 for the no-trans and trans data, respectively). However, the angle responses were systematically underpredicted. We therefore do not consider the model further.

3. An alternative argument might be that the location bias occurred because subjects were simply moving toward a comfortable, central resting point of the arm. However, the finding that errors did not differ according to whether a single central point was used for all paths (the 1-starting-point condition) or whether the starting point varied (the 10starting-point condition) suggests that subjects were not biased toward a single spatial location on the grounds of postural comfort.

4 . This tendency for angular errors to increase with distance from the origin is contrary to what one would expect from purely geometric constraints: A given distance in arc length translates into a smaller angular measure, the larger the radius. If subjects tend to err a constant arc distance from the origin, their angular errors would decrease with the distance along the observed response leg.

(Manuscript received September 12, 1997; revision accepted for publication February 13, 1998.) 